Supporting Information

\title{
Kinetic Model of Calcium-Silicate Hydrate Nucleation and Growth in the Presence of PCE Superplasticizers
}

Luca Valentini*1, Marco Favero ${ }^{1}$, Maria C. Dalconi ${ }^{1}$, Vincenzo Russo ${ }^{2}$, Giorgio Ferrari $^{2}$, and Gilberto Artioli ${ }^{1}$

${ }^{1}$ Department of Geosciences and CIRCe Center, University of Padua, Padua, Italy

${ }^{2}$ Research and Development Department, Mapei SpA, Milan, Italy 


\section{Sample preparation and XRD measurements}

Laboratory-quality monoclinic tricalcium silicate $\left(\mathrm{C}_{3} \mathrm{~S}\right)$ has been used for the in-situ X-ray powder diffraction (XRD) measurements. A XRD diffraction profile of a dry sample is displayed in Figure S1. The BET surface area measured by nitrogen adsorption was $423.6 \mathrm{~m}^{2} / \mathrm{kg}$. The particle size distribution, measured by laser diffraction, is displayed in Figure S2.

Pastes have been prepared by mixing $\mathrm{C}_{3} \mathrm{~S}$, deionized water and the needed amount of $\mathrm{PCE}$

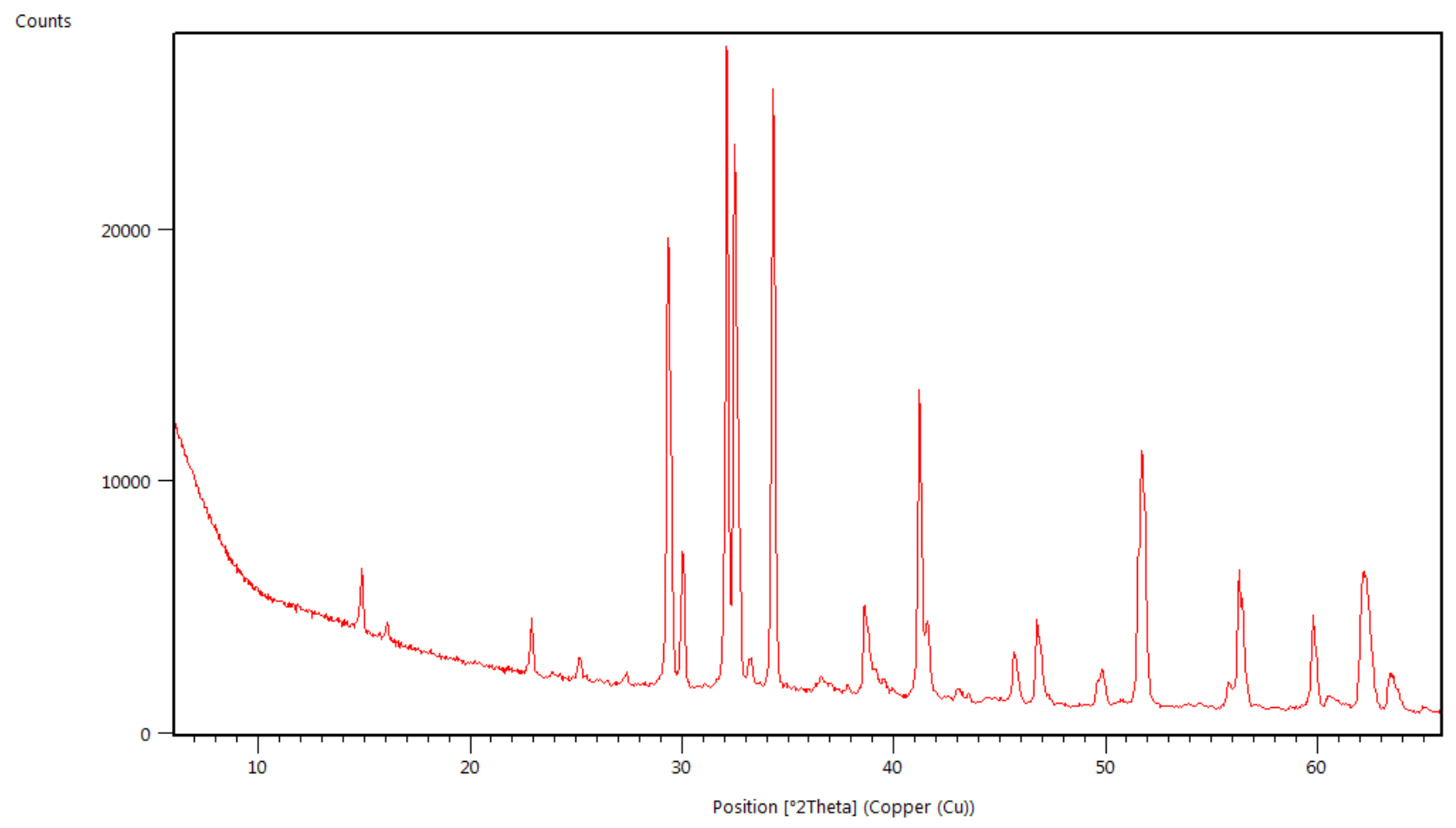

Figure S 1: Diffraction profile of the dry $\mathrm{C}_{3} \mathrm{~S}$ used for the experiments.

superplasticizer, using an orbital stirrer, with a mixing time of five minutes. In the first set of experiments, the PCEs were added directly to the mix water, before mixing. In the second set of experiments, the PCEs were added with a delay of four minutes, after initial mixing of the $\mathrm{C}_{3} \mathrm{~S}$ powder with deionized water. XRD measurements have been performed in focusing transmission capillary geometry with an X'Pert PRO MPD instrument (PANalytical, Almelo, the Netherlands), mounting a $\mathrm{Cu}$ LFF anode. The incident beam was focused by a multi-layered W/Si crystal to give no diffraction lines, to select the incident wavelength and reducing the $\mathrm{K} \beta$ contribution and 


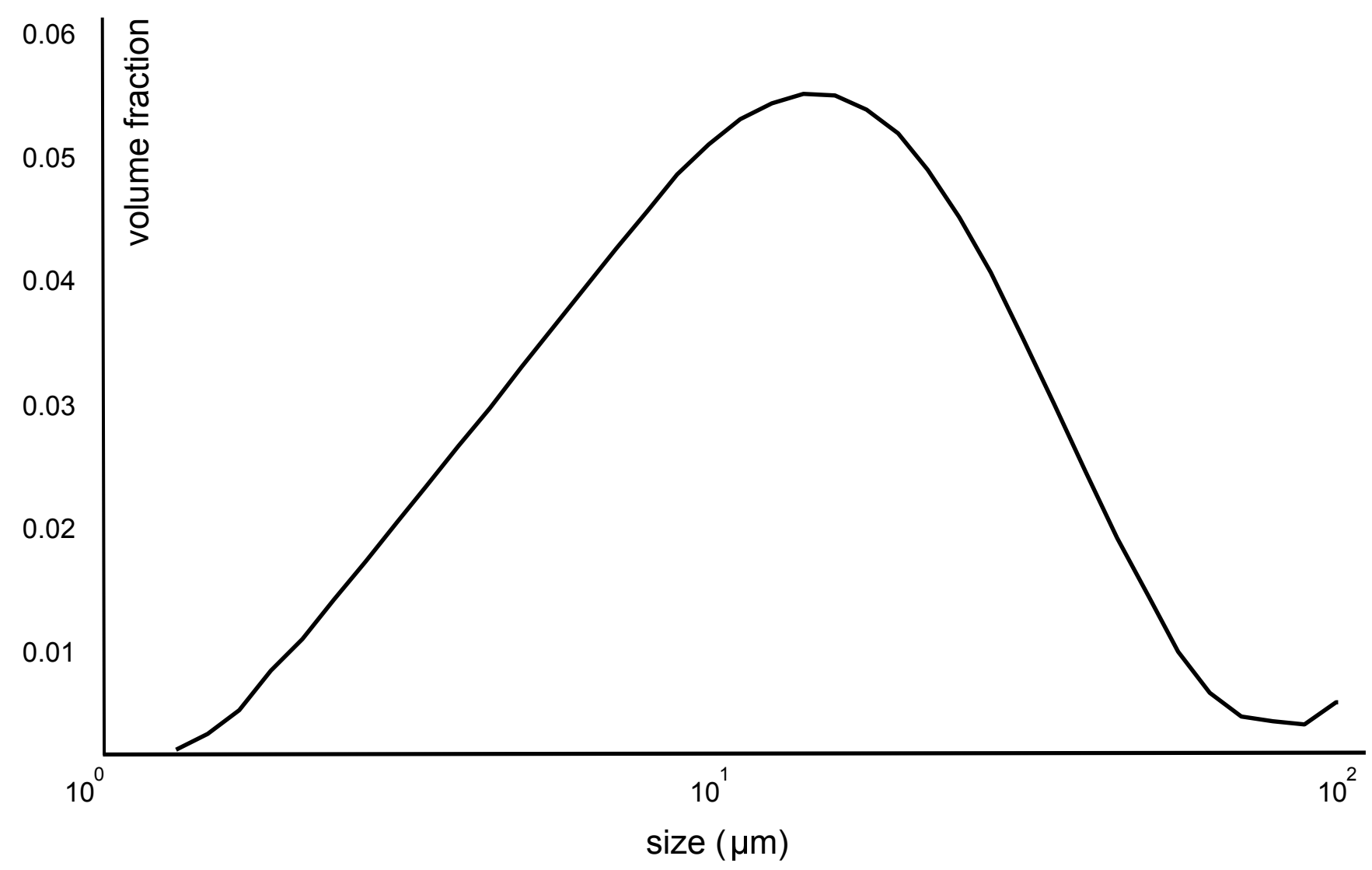

Figure $\mathrm{S} 2: \mathrm{C}_{3} \mathrm{~S}$ particle size distribution.

thus avoiding the use of a $\mathrm{Ni}$ filter. Boron-glass capillaries, having a nominal diameter of 0.5 $\mathrm{mm}$, were filled with the $\mathrm{C}_{3} \mathrm{~S}$ pastes and immediately mounted into the diffractometer. In-situ kinetics were measured for approximately 24 hours (20 minutes per scan) from $3^{\circ}$ to $66^{\circ} 2 \theta$, with a step size of $0.026^{\circ} 2 \theta$ and a count time per step of $120 \mathrm{~s}$. The temperature inside the diffractometer case was set to $23.5^{\circ} \mathrm{C} \pm 0.5^{\circ} \mathrm{C}$. Divergence, antiscatter $\left(0.5^{\circ}\right)$ and Soller slits $(0.04$ rad) were implemented in the setup. Semi-quantitative phase analysis of collected patterns have been performed using the Bruker Topas v.4.1 software (Bruker, Billerica, USA), adjusting the instrumental parameters on a NIST SRM 640c (Silicon) standard previously measured in the same instrumental conditions. The refinement procedure included background approximation using a seventh order Chebyshev polynomial, specimen displacement for Debye-Scherrer geometry and linear absorption correction. For each phase, unit cell parameters and the Lorentian component 
of crystallite size were refined independently. The scale factor is the only parameter that was refined in the whole procedure. No crystal structure parameter has been considered during the refinement procedure. Bragg peaks contributions of $\mathrm{C}_{3} \mathrm{~S}$ were described by the triclinic structural model of Golovastikov et $\mathrm{al}^{\mathrm{S} 1}$. whereas the structural model reported in the Topas cement structure database was used for portlandite.

\section{PCE characterization}

Molecular weight distribution analysis (MWD) was carried out with a gel permeation chromatography (GPC) apparatus consisting of a Waters 515 pump (1 ml/min) equipped with a Waters 717 Plus auto-sampler. Separation was performed by a set of four Waters Ultrahydrogel columns (7.8x3000 mm, pore size 2x120 A, $250 \AA, 500 \AA)$. The detection was performed by a Waters 410 Differential Refractometer detector (temperature $40^{\circ} \mathrm{C}$ ). The data were evaluated using the Waters Empower Pro software. All samples were dissolved in the mobile phase (NaNO3 0.04M 80\% - Acetonitrile $20 \%$ ) at $1 \%$ concentration. The molecular weight distribution of the analyzed polymers was determined by using a calibration curve obtained with standards polysaccharides (Polymer Laboratories LTD). The charge density of the different PCEs, expressed as the ratio of carboxylic groups/ester groups in the backbone, was determined by potentiometric titration, carried out with an acid/base titration with $\mathrm{HCl} 0.1 \mathrm{M}$ performed in alkali media with the automatic titration system Mettler Toledo T70 equipped with a Rondo20 autosampler.

(S1) Golovastikov, N. I; Matveeva, R. G; Belov, N. V. Kristallografiya 1975, 20, 721-729. 